\title{
Electric-Elastic Field Induced by a Straight Dislocation in One-Dimensional Quasicrystals
}

\author{
L.-Z. YANG ${ }^{a, b}, \mathrm{Y} . \mathrm{GAO}^{a, *}, \mathrm{E} \mathrm{PAN}^{c}$ AND N. WAKSMANSKI ${ }^{c}$ \\ ${ }^{a}$ College of Science, China Agricultural University, Beijing 100083, China \\ ${ }^{b}$ College of Engineering, China Agricultural University, Beijing 100083, China \\ ${ }^{c}$ Department of Civil Engineering, University of Akron, Akron, OH 44325-3905, USA
}

\begin{abstract}
By using the generalized Stroh formalism, the electric-elastic field induced by a straight dislocation parallel to a periodic axis of a one-dimensional quasicrystal is obtained. The derivation is concise and the solution is in an exact closed form. As an illustration, the electric-elastic fields around a straight dislocation in a one-dimensional hexagonal quasicrystal are studied. Besides the interesting numerical results presented, the generalized Stroh formalism can be applied to more complicated dislocation problems in quasicrystals.
\end{abstract}

DOI: 10.12693/APhysPolA.126.467

PACS: 61.44.Br, 61.72.Lk, 62.20.D-

\section{Introduction}

Since the discovery of quasicrystals (QCs) around 1982 [1], structural, electronic, magnetic, thermal and mechanical properties of the QC matter have been investigated intensively. In QCs, there is not only a phonon field which is related to the translations of atoms (standard elasticity), but also a phason field which is related to rearrangements of atomic configurations. The elasticity of QCs is described by the generalized elastic theory established by Ding et al. [2] that has been proved to be a powerful and important tool to study the mechanical behavior of QCs. Expressions of physical properties of QCs, such as elasticity, thermal expansion, and piezoelectricity tensors have been obtained in [3] and [4].

Studies of dislocations in QCs have attracted extensive attention because of their importance not only in QC structural studies but also in understanding many of their physical and mechanical properties. So far, analytical expressions for dislocation-induced elastic fields in many QCs have been derived by methods including Green's function method [5], Stroh eigenvalue method [6], Eshelby method [7], and displacement function method [8]. Up to now, however, analytical solutions of dislocations in piezoelectric QCs have not been reported in literature.

This paper utilizes the generalized Stroh formalism to obtain the electric-elastic field induced by a straight dislocation which is parallel to a periodic axis of a one-dimensional (1D) hexagonal QC. As an illustration, numerical results of the induced electric-elastic field are presented and analyzed.

\section{Basic equations}

A 1D QC is defined as a three-dimensional body where its atom arrangement is quasi-periodic in the $x_{3}$-axis and periodic in the $x_{1}-x_{2}$ plane referred to a coordinate system $\left(x_{1}, x_{2}, x_{3}\right)$. The polarized direction in the $\mathrm{QC}$ is assumed along the $x_{3}$-axis.

*corresponding author; e-mail: gaoyangg@gmail.com
Within the theory of elasticity of 1D QCs [2], displacements are denoted as $u_{i}(i=1,2,3)$ in the phonon field and $w_{3}$ in the phason field, both of which depend on the coordinate in real space. The linear electric-elastic coupled constitutive equations [4] of the 1D QCs can be written as

$$
\begin{aligned}
& \sigma_{i j}=C_{i j k l} \varepsilon_{k l}+d_{i j k} \omega_{k}-e_{i j k}^{(1)} E_{k}, \\
& H_{3 j}=d_{k l j} \varepsilon_{k l}+K_{j k} \omega_{k}-e_{3 j k}^{(2)} E_{k}, \\
& D_{j}=e_{k l j}^{(1)} \varepsilon_{k l}+e_{k 3 j}^{(2)} \omega_{k}+\kappa_{j k} E_{k},
\end{aligned}
$$

where

$$
\varepsilon_{i j}=\left(u_{i, j}+u_{j, i}\right) / 2, \quad \omega_{j}=w_{3, j}, \quad E_{j}=-\varphi, j,
$$

$j, k, l=1,2,3$, repeated indices imply summation and a comma stands for differentiation. $C_{i j k l}$ and $K_{j k}$ denote the elastic constants in phonon and phason fields, respectively, $d_{i j k}$ - the phonon-phason coupling elastic constants, $e_{i j k}^{(1)}$ and $e_{3 j k}^{(2)}$ - the piezoelectric constants in phonon and phason fields, respectively, $\kappa_{j k}$ - the permittivity constants, and $\varphi$ - the electric potential. In the following formulation, lowercase subscripts will always range from 1 to 3 , and uppercase subscripts from 1 to 5 .

In the absence of body sources, the equilibrium equations in the piezoelectric QC materials are

$$
\sigma_{i j, j}=0, \quad H_{3 j, j}=0, \quad D_{j, j}=0,
$$

where $\sigma_{i j}, H_{3 j}$ and $D_{j}$ denote, respectively, the phonon stresses, phason stresses, and electric displacements.

Following the same approach as in magneto-electro-elastic media [9], the basic Eqs. (2.1) to (2.3) can be combined by using the generalized field quantities. First, we introduce the generalized displacement vector as

$$
\boldsymbol{u}=\left\{u_{1}, u_{2}, u_{3}, w_{3}, \varphi\right\}^{\mathrm{T}},
$$

where the superscript "T" stands for the matrix transpose. Then, we defined the $5 \times 3$ generalized stress tensor $\sigma_{I j}(I=1,2,3,4,5)$ as

$$
\sigma_{I j}=\sigma_{i j} \quad(I=1,2,3), \quad \sigma_{4 j}=H_{3 j}, \quad \sigma_{5 j}=D_{j} .
$$

Finally, the generalized material properties are defined as: 


$$
\begin{aligned}
& E_{i j k l}=C_{i j k l}, \quad E_{i j 4 l}=d_{i j l}, \quad E_{i j 5 l}=e_{i j l}^{(1)} \text {; } \\
& E_{4 j k l}=d_{k l j}, \quad E_{4 j 4 l}=K_{j l}, \quad E_{4 j 5 l}=e_{3 j l}^{(2)} \text {; } \\
& E_{5 j k l}=e_{k l j}^{(1)}, \quad E_{5 j 4 l}=e_{l 3 j}^{(2)}, \quad E_{5 j 5 l}=-\kappa_{j l} \text {. }
\end{aligned}
$$

With these generalized quantities, Eqs. (2.1) and (2.3) can be rewritten uniformly as

$$
\begin{aligned}
& \sigma_{i j}=E_{i j k l} u_{k, l}, \\
& \sigma_{i j, j}=0 .
\end{aligned}
$$

3. Generalized Stroh formalism for $1 \mathrm{D}$ QCs

It is well known that the Stroh formalism [10-12] is powerful and convenient in dealing with plane deformations of anisotropic elastic materials. In this section, the Stroh formalism for elastic/piezoelectric solids will be extended to $1 \mathrm{D}$ QC solids.

We assume that the dislocation is infinite long along $x_{2}$ direction so that the generalized stresses and displacements are independent of the $x_{2}$-axis. Since all field variables are only dependent on $x_{1}$ and $x_{3}$, Eq. (2.8) can be rewritten as

$$
\sigma_{I 1,1}+\sigma_{I 3,3}=0 .
$$

From Eq. (3.1) it can be seen that there exists a generalized stress function vector $\phi$ representing the stress components $\sigma_{I 1}$ and $\sigma_{I 3}$ as

$$
\sigma_{I 1}=-\phi_{I, 3}, \quad \sigma_{I 3}=\phi_{I, 1} \text {. }
$$

By virtue of the constitutive relation Eq. (2.7), Eq. (3.2) can be transformed into

$$
Q u_{, 1}+R u_{, 3}=-\phi_{, 3}, \quad R^{\mathrm{T}} u_{, 1}+T u_{, 3}=\phi_{, 1} .
$$

where $Q, R$, and $T$ are $5 \times 5$ real matrices defined by the material constants as

$$
Q_{I K}=E_{I 1 K 1}, \quad R_{I K}=E_{I 1 K 3}, \quad T_{I K}=E_{I 3 K 3} .
$$

The general solutions for $u$ and $\phi$ can be assumed as

$$
u=a f(z), \quad \phi=b f(z),
$$

where $z=x_{1}+p x_{3}, f$ is an arbitrary complex function of $z, a$ and $b$ are unknown vectors and $p$ is the eigenvalue, to be given below. Inserting Eq. (3.5) into Eq. (3.3), the relation between $b$ and $a$ is found to be

$$
b=\left(R^{\mathrm{T}}+p R\right) a=-p^{-1}(Q+p R) a .
$$

Then, using Eq. (3.6), Eq. (3.1) can be recast into a $10 \times 10$ linear eigensystem

$$
N \xi=p \xi, \quad \xi=\{a, b\}^{\mathrm{T}},
$$

where

$$
N=\left[\begin{array}{lr}
-T^{-1} R^{\mathrm{T}} & T^{-1} \\
-Q+R T^{-1} R^{\mathrm{T}} & -R T^{-1}
\end{array}\right] .
$$

A nontrivial solution for $\xi$ exists if the determinate of the characteristic matrix in Eq. (3.7) is zero. In other words,

$$
\operatorname{det}(N-p I)=0 \text {, }
$$

where $I$ is a $5 \times 5$ unit matrix. The ten eigenvalues $p$ and the corresponding eigenvectors $\xi$ in Eq. (3.7) are determined by Eq. (3.9). The fact that the generalized strain energy should always be positive requires that the eigenvalues $p$ should be complex [10]. Since the coefficients of Eq. (3.7) are real, there are five pairs of $p$, complex conjugate to each other. If $p_{I}$ denotes the eigenvalues, $a_{I}$ and $b_{I}$ are the eigenvectors of Eq. (3.7), we can let

$$
p_{I+5}=\bar{p}_{I}, \quad \operatorname{Im}\left(p_{I}\right)>0, \quad a_{I+5}=\bar{a}_{I}, \quad b_{I+5}=\bar{b}_{I},
$$

where "Im" represents the imaginary part and the overbar denotes the conjugate of the quantity. Assuming that all eigenvalues are distinct the general solution for the displacement and stress function vectors in Eq. (3.5) are derived as

$$
\left(\begin{array}{l}
u \\
\phi
\end{array}\right)=\left[\begin{array}{ll}
A & \bar{A} \\
B & \bar{B}
\end{array}\right]\left(\begin{array}{l}
f \\
\bar{f}
\end{array}\right),
$$

where

$$
\begin{aligned}
& A=\left[a_{1}, a_{2}, a_{3}, a_{4}, a_{5}\right], \quad B=\left[b_{1}, b_{2}, b_{3}, b_{4}, b_{5}\right], \\
& f(z)=\left[f\left(z_{1}\right), f\left(z_{2}\right), f\left(z_{3}\right), f\left(z_{4}\right), f\left(z_{5}\right)\right]^{\mathrm{T}} .
\end{aligned}
$$

The eigenvector matrices $A$ and $B$ satisfy the following normalization relation:

$$
B^{\mathrm{T}} A+A^{\mathrm{T}} B=I \text {. }
$$

$\mathrm{Up}$ to this point, the dislocation problem in $1 \mathrm{D} \mathrm{QCs}$ reduces to the determination of the complex vector function $f$ under certain given boundary conditions. This is done below for the given dislocation problem. However, before presenting our numerical example, we introduce the following three important matrices:

$$
S=\mathrm{i}\left(2 A B^{\mathrm{T}}-I\right), \quad H=2 \mathrm{i} A A^{\mathrm{T}}, \quad L=-2 \mathrm{i} B B^{\mathrm{T}} .
$$

We remark that, by following the same approach in elastic and piezoelectric media $[12,13]$, these three matrices are all real.

\section{Generalized solution for a straight dislocation in a 1D $\mathrm{QC}$}

The Burgers vector in 1D QC is defined as $\hat{b}^{\|} \oplus \hat{b}^{\perp}=$ $\left\{b_{1}^{\|}, b_{2}^{\|}, b_{3}^{\|}, b_{3}^{\perp}\right\}$. To analyze the electric-elastic field in the $1 \mathrm{D} \mathrm{QC}$, we extend the Burgers vector definition as

$$
\hat{b}=\left\{b_{1}^{\|}, b_{2}^{\|}, b_{3}^{\|}, b_{3}^{\perp}, b^{\varphi}\right\} .
$$

Considering a dislocation along $x_{2}$-axis with the Burgers vector $\hat{b}$ in a $1 \mathrm{D} \mathrm{QC}$ with the core at the origin far from the boundary of an infinite space, the boundary conditions of this problem can be written as

$$
\begin{aligned}
& \oint_{\Gamma} \mathrm{d} \phi=0 \text { for any closed curve } \Gamma \\
& \oint_{\Gamma} \mathrm{d} u=\hat{b} \text { for any closed } \Gamma \text { enclosing the origin, } \\
& \sigma_{I j} \rightarrow 0 \text { at infinity. }
\end{aligned}
$$

Extending the solutions for dislocation problems in elastic and piezoelectric materials $[12,13]$ to $1 \mathrm{D}$ QC, the closed-form solutions for the displacement vector, stress function vector can then be found as

$$
\begin{aligned}
u & =-\frac{1}{2 \pi}[(\ln r) S+\pi(S(\theta) S-H(\theta) L)] \hat{b}, \\
\phi & =\frac{1}{2 \pi}\left[(\ln r) L+\pi\left(L(\theta) L-S^{\mathrm{T}}(\theta) S\right)\right] \hat{b},
\end{aligned}
$$

in which $r=x_{1}^{2}+x_{3}^{2}, \theta$ is the angle so that $x_{1}=r \cos \theta$ and $x_{3}=r \sin \theta$. The matrices $S, H, L$ in Eq. (3.14) can now be expressed as functions of $\theta$, as $S(\theta), H(\theta), L(\theta)$ which are defined as:

$$
S(\theta)=\frac{2}{\pi} \operatorname{Re}\left[A\left\langle\ln \left(\cos \theta+p_{*} \sin \theta\right)\right\rangle B^{\mathrm{T}}\right],
$$




$$
\begin{aligned}
& H(\theta)=\frac{2}{\pi} \operatorname{Re}\left[A\left\langle\ln \left(\cos \theta+p_{*} \sin \theta\right)\right\rangle A^{\mathrm{T}}\right], \\
& L(\theta)=\frac{2}{\pi} \operatorname{Re}\left[B\left\langle\ln \left(\cos \theta+p_{*} \sin \theta\right)\right\rangle B^{\mathrm{T}}\right] .
\end{aligned}
$$

In Eq. (4.5) $p_{*}$ is defined as:

$$
p_{*}=(p \cos \theta-\sin \theta) /(p \sin \theta+\cos \theta) \text {. }
$$

We introduce five direction vectors as

$$
\begin{aligned}
& m^{\mathrm{T}}=\{\cos \theta, 0, \sin \theta, 0,0\}, \\
& n^{\mathrm{T}}=\{-\sin \theta, 0, \cos \theta, 0,0\}, \quad i_{3}^{\mathrm{T}}=\{0,1,0,0,0\}, \\
& i_{4}^{\mathrm{T}}=\{0,0,0,1,0\}, \quad i_{5}^{\mathrm{T}}=\{0,0,0,0,1\} .
\end{aligned}
$$

Then, the stresses and electric displacement in the polar coordinate system due to the concentrated dislocation can be expressed as

$$
\begin{aligned}
& \sigma_{r r}=\frac{1}{2 r} m^{\mathrm{T}}\left(N_{3}(\theta) S-N_{1}^{\mathrm{T}}(\theta) L\right) \hat{b}, \quad \sigma_{\theta \theta}=\frac{1}{2 \pi r} n^{\mathrm{T}} L b, \\
& \sigma_{r \theta}=\frac{1}{2 \pi r} m^{\mathrm{T}} L \hat{b}, \quad \sigma_{r 2}=\frac{1}{2 r} i_{3}^{\mathrm{T}}\left(N_{3}(\theta) S-N_{1}^{\mathrm{T}}(\theta) L\right) \hat{b}, \\
& \sigma_{\theta 2}=\frac{1}{2 \pi r} i_{3}^{\mathrm{T}} L \hat{b}, \quad H_{3 r}=\frac{1}{2 r} i_{4}^{\mathrm{T}}\left(N_{3}(\theta) S-N_{1}^{\mathrm{T}}(\theta) L\right) \hat{b}, \\
& H_{3 \theta}=\frac{1}{2 \pi r} i_{4}^{\mathrm{T}} L \hat{b}, \quad D_{r}=\frac{1}{2 r} i_{5}^{\mathrm{T}}\left(N_{3}(\theta) S-N_{1}^{\mathrm{T}}(\theta) L\right) \hat{b}, \\
& D_{\theta}=\frac{1}{2 \pi r} i_{5}^{\mathrm{T}} L \hat{b},
\end{aligned}
$$

where

$$
\begin{aligned}
& N_{1}(\theta)=-T^{-1}(\theta) R^{\mathrm{T}}(\theta), \\
& N_{3}(\theta)=-R(\theta) T^{-1}(\theta) R^{\mathrm{T}}(\theta)-Q(\theta), \\
& Q(\theta)=Q \cos ^{2} \theta+\left(R+R^{\mathrm{T}}\right) \sin \theta \cos \theta+T \sin ^{2} \theta, \\
& R(\theta)=R \cos ^{2} \theta+(T-Q) \sin \theta \cos \theta-R^{\mathrm{T}} \sin ^{2} \theta, \\
& T(\theta)=T \cos ^{2} \theta-\left(R+R^{\mathrm{T}}\right) \sin \theta \cos \theta+Q \sin ^{2} \theta .
\end{aligned}
$$

The expressions for the remaining stress components $\sigma_{22}$, $H_{32}$, and $D_{2}$ can be found from the following three conditions:

$$
\varepsilon_{22}=0, \quad \omega_{2}=0, \quad E_{2}=0 .
$$

\section{Electric-elastic field for a straight dislocation in a 1D hexagonal QC}

For 1D hexagonal QCs, the linear constitutive equations take the following form [3]:

$$
\begin{aligned}
& \sigma_{11}=C_{11} \varepsilon_{11}+C_{12} \varepsilon_{22}+C_{13} \varepsilon_{33}+R_{1} w_{33}-e_{31}^{(1)} E_{3}, \\
& \sigma_{22}=C_{12} \varepsilon_{11}+C_{11} \varepsilon_{22}+C_{13} \varepsilon_{33}+R_{1} w_{33}-e_{31}^{(1)} E_{3}, \\
& \sigma_{33}=C_{13} \varepsilon_{11}+C_{13} \varepsilon_{22}+C_{33} \varepsilon_{33}+R_{2} w_{33}-e_{33}^{(1)} E_{3}, \\
& \sigma_{23}=\sigma_{32}=2 C_{44} \varepsilon_{23}+R_{3} w_{32}-e_{15}^{(1)} E_{2}, \\
& \sigma_{31}=\sigma_{13}=2 C_{44} \varepsilon_{13}+R_{3} w_{31}-e_{15}^{(1)} E_{1}, \\
& \sigma_{12}=\sigma_{21}=2 C_{66} \varepsilon_{12}, \\
& H_{33}=2 R_{1} \varepsilon_{12}+R_{2} \varepsilon_{33}+K_{1} w_{33}-e_{33}^{(2)} E_{3}, \\
& H_{32}=2 R_{3} \varepsilon_{23}+K_{2} w_{32}-e_{15}^{(2)} E_{2}, \\
& H_{31}=2 R_{3} \varepsilon_{13}+K_{2} w_{31}-e_{15}^{(2)} E_{1}, \\
& D_{3}=2 e_{31}^{(1)} \varepsilon_{12}+e_{33}^{(1)} \varepsilon_{33}+e_{33}^{(2)} w_{33}+\kappa_{33} E_{3}, \\
& D_{2}=2 e_{15}^{(1)} \varepsilon_{23}+e_{15}^{(2)} w_{32}+\kappa_{11} E_{2}, \\
& D_{1}=2 e_{15}^{(1)} \varepsilon_{13}+e_{15}^{(2)} w_{31}+\kappa_{11} E_{1} .
\end{aligned}
$$

According to Eqs. (2.1), (2.6), (3.4) and (5.1), the matrices $Q, R$, and $T$ in the Stroh formalism can be determined as

$$
\begin{aligned}
Q & =\left[\begin{array}{ccccc}
C_{11} & 0 & 0 & 0 & 0 \\
0 & C_{66} & 0 & 0 & 0 \\
0 & 0 & C_{44} & R_{3} & e_{15}^{(1)} \\
0 & 0 & R_{3} & K_{2} & e_{15}^{(2)} \\
0 & 0 & e_{15}^{(1)} & e_{15}^{(2)} & -\kappa_{11}
\end{array}\right], \\
R & =\left[\begin{array}{ccccc}
0 & 0 & C_{13} & R_{1} & e_{31}^{(1)} \\
0 & 0 & 0 & 0 & 0 \\
C_{44} & 0 & 0 & 0 & 0 \\
R_{3} & 0 & 0 & 0 & 0 \\
e_{15}^{(1)} & 0 & 0 & 0 & 0
\end{array}\right], \\
T & =\left[\begin{array}{ccccc}
C_{44} & 0 & 0 & 0 & 0 \\
0 & C_{44} & 0 & 0 & 0 \\
0 & 0 & C_{33} & R_{2} & e_{33}^{(1)} \\
0 & 0 & R_{2} & K_{1} & e_{33}^{(2)} \\
0 & 0 & e_{33}^{(1)} & e_{33}^{(2)} & -\kappa_{33}
\end{array}\right] .
\end{aligned}
$$

According to the material constants in QCs [14] and piezoelectric crystals [15], the material constants in Eq. (5.2) for a 1D hexagonal QC are assumed as $C_{11}=$ $23.433 \times 10^{10} \mathrm{~N} / \mathrm{m}^{2}, C_{12}=5.741 \times 10^{10} \mathrm{~N} / \mathrm{m}^{2}, C_{13}=$ $6.663 \times 10^{10} \mathrm{~N} / \mathrm{m}^{2}, C_{33}=23.222 \times 10^{10} \mathrm{~N} / \mathrm{m}^{2}, C_{44}=$ $7.019 \times 10^{10} \mathrm{~N} / \mathrm{m}^{2}, C_{66}=\left(C_{11}-C_{12}\right) / 2=8.846 \times$ $10^{10} \mathrm{~N} / \mathrm{m}^{2}, R_{1}=R_{2}=R_{3}=8.846 \times 10^{8} \mathrm{~N} / \mathrm{m}^{2}$, $K_{1}=12.2 \times 10^{10} \mathrm{~N} / \mathrm{m}^{2}, K_{2}=2.4 \times 10^{10} \mathrm{~N} / \mathrm{m}^{2}, e_{15}^{(1)}=$ $11.6 \mathrm{C} / \mathrm{m}^{2}, e_{31}^{(1)}=-4.4 \mathrm{C} / \mathrm{m}^{2}, e_{33}^{(1)}=18.6 \mathrm{C} / \mathrm{m}^{2}, e_{15}^{(2)}=$ $1.16 \mathrm{C} / \mathrm{m}^{2}, e_{33}^{(2)}=1.86 \mathrm{C} / \mathrm{m}^{2}, \kappa_{11}=5 \times 10^{-9} \mathrm{C}^{2} /\left(\mathrm{N} \mathrm{m}^{2}\right)$, $k_{33}=10 \times 10^{-9} \mathrm{C}^{2} /\left(\mathrm{N} \mathrm{m}^{2}\right)$.

The electric-elastic field induced by a straight dislocation along $x_{2}$ axis of a $1 \mathrm{D}$ hexagonal QC is investigated. The Burgers vector is assumed as $\hat{b}^{\prime}=\{a, 0,0,0,0\}$. The contours of the generalized displacements around the dislocation in the $x_{1}-x_{3}$ plane are shown in Fig. 1 . It is clearly observed that these contours are either symmetric or antisymmetric about the coordinate axes, which is consistent with the hexagonal feature of the 1D QCs.

Figure 2 presents the contours of the generalized stresses around the dislocation in $x_{1}-x_{3}$ plane. It is obvious that these stresses decrease with increasing distance from the origin of the dislocation. Compared to Fig. 1 for the generalized displacements, we also observe that while the stresses are also either symmetric or antisymmetric about the coordinate axes, the contour curves in the stresses are more complicated than those in the displacements.

From Figs. 1 and 2, it can be seen that the dislocation in phonon field has great influences on the displacements and stresses in the phason field and electric field. The phonon, phason, and electric fields are all coupled together. We also mention that many other cases of dislocations in 1D QC can be calculated by simply chang- 


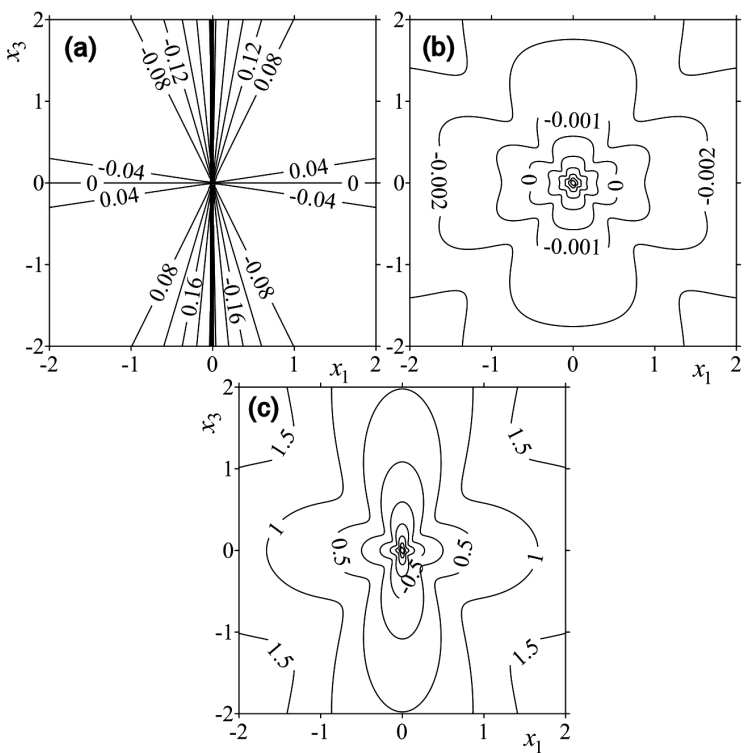

Fig. 1. The generalized displcements induced by a straight dislocation with Burgers vector $\hat{b}^{\prime}$ : (a) $u_{1} / a$, (b) $w_{3} / a$, (c) $\varphi / a$ (unit: $10^{8} \mathrm{~N} / \mathrm{C}$ ).

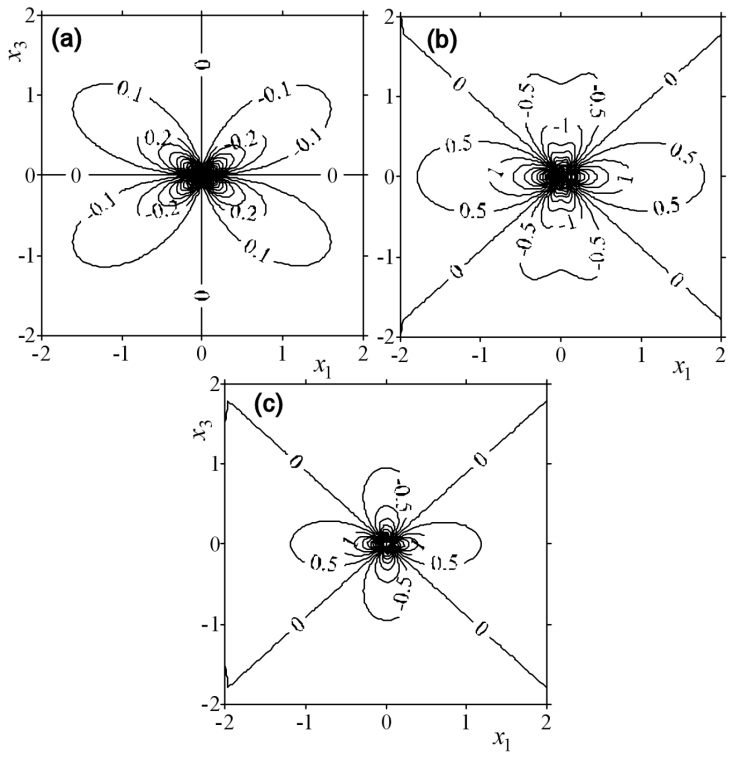

Fig. 2. The generalized stresses induced by a straight dislocation with Burgers vector $\hat{b}^{\prime}$ (unit: $1 / m$ ): (a) $\sigma_{r r} /\left(a C_{11}\right)$, (b) $H_{3 r} /\left(a R_{1}\right)$, (c) $D_{r} /\left(a e_{15}^{(1)}\right)$.

ing the components of the Burgers vector $\hat{b}$ in Eqs. (4.4) and (4.8).

\section{Conclusions}

Based on the generalized Stroh formalism, the electric-elastic field induced by the straight dislocation parallel to a periodic axis of a $1 \mathrm{D} \mathrm{QC}$ is given in this paper. The derivation is simple and straightforward so that the generalized displacements and stresses can be easily calculated. As a numerical example, the electric-elastic field in a 1D hexagonal QC due to a straight dislocation is investigated. The results show clearly the coupling effects among the phonon, phason and electric fields. We also mention that the Stroh formalism can also be extended to the more complicated dislocation problems in other QCs.

\section{Acknowledgments}

The work is supported by the National Natural Science Foundation of China (No. 11172319), Chinese Universities Scientific Fund (No. 2011JS046 and No. 2013BH008), Program for New Century Excellent Talents in University, Opening Fund of State Key Laboratory of Nonlinear Mechanics, and National Science Foundation for Post-doctoral Scientists of China.

\section{References}

[1] D. Shechtman, I. Blech, D. Gratias, J.W. Cahn, Phys. Rev. Lett. 53, 1951 (1984).

[2] D.H. Ding, W.G. Yang, C.Z. Hu, R.H. Wang, Phys. Rev. B 48, 7003 (1993).

[3] C.L. Lian, Y.Y. Liu, Chin. Phys. 13, 924 (2004).

[4] C.Z. Hu, R.H. Wang, D.H. Ding, Rep. Prog. Phys. 63, 1 (2000).

[5] D.H. Ding, R.H. Wang, W.G. Yang, C.Z. Hu, J. Phys., Condens. Matter 7, 5423 (1995).

[6] D.H. Ding, R.H. Wang, W.G. Yang, C.Z. Hu, Y.L. Qin, Philos. Mag. 72, 353 (1995).

[7] Y.L. Qin, R H. Wang, D.H. Ding, J.L. Lei, J. Phys., Condens. Matter 9, 859 (1997).

[8] X.F. Li, X.Y. Duan, T.Y. Fan, Y.F. Sun, J. Phys., Condens. Matter 11, 703 (1999).

[9] E. Pan, J. Appl. Mech. 68, 608 (2001); A.N. Stroh, Philos Mag. 3, 625 (1958).

[10] A.N. Stroh, Philos. Mag. 3, 625 (1958).

[11] A.N. Stroh, J. Math. Phys. 41, 77 (1962).

[12] T.C.T. Ting, Anisotropic Elasticity. Theory and Applications, Oxford University Press, New York 1996.

[13] C. Hwu, Anisotropic Elastic Plates, Springer, New York 2010

[14] T.Y. Fan, Engineering 5, 407 (2013).

[15] J.S. Lee, L.Z. Jiang, Int. J. Solids Struct. 33, 977 (1996). 